\title{
SOPORTE NUTRICIONAL EN EL PACIENTE PEDIÁTRICO CRÍTICO
}

\author{
NUTRITIONAL SUPPORT IN THE CRITICAL PEDIATRIC PATIENT \\ Liliana Paola Cieza-Yamunaqué1
}

\begin{abstract}
RESUMEN
El soporte nutricional del paciente pediátrico crítico influencia directamente en su morbimotalidad. Diversas condiciones hacen al niño particularmente vulnerable a la desnutrición cuando tiene enfermedades graves. La respuesta metabólica al estrés es principalmente hipercatabolismo de proteínas que ocasiona un balance nitrogenado negativo. Es importante adoptar estrategias que permitan proporcionar una adecuada y oportuna nutrición, realizando una buena valoración nutricional, calculando el gasto de los requerimientos energéticos, priorizando el aporte de proteínas como el macronutriente de mayor relevancia, iniciando el soporte nutricional temprano, mediante la vía correcta y realizando el monitoreo y seguimiento oportuno. En conclusión, el soporte nutricional debe formar parte importante de la estrategia terapéutica en todo niño crítico.
\end{abstract}

Palabras clave: Unidad de cuidados intensivos pediátricos; Requerimientos nutricionales; Nutrición enteral; Nutrición parenteral. (fuente: DeCS BIREME)

\begin{abstract}
The nutritional support of the critical pediatric patient directly influences their morbimotality. Various conditions make the child vulnerable to malnutrition when he has serious illnesses. The metabolic response to stress is mainly protein hypercatabolism that causes a negative nitrogen balance. It is important that it suits you as a source of energy, to be carried out in the future. Performing the monitoring and timely follow-up. In conclusion, nutritional support should be an important part of the therapeutic strategy in every critical child.
\end{abstract}

Key words: Pediatric intensive care unit; Nutritional requirements; Enteral nutrition; Parenteral nutrition. (source: MeSH NLM)

\section{INTRODUCCIÓN}

El soporte nutricional del paciente pediátrico crítico es de suma importancia en el manejo de su enfermedad, influenciando directamente en la morbimotalidad. En el niño crítico diversas condiciones lo hacen particularmente vulnerable a la desnutrición y alta mortalidad debido a la falla en el soporte nutricional. En primer lugar el mismo proceso inflamatorio que ocasiona una respuesta metabólica principalmente caracterizada por gran pérdida proteica e hipercatabolismo, en segundo lugar el ayuno que puede venir presentado o presentar durante su hospitalización, aumenta la noxa y además la menor reserva de proteínas y grasa que tienen los niños predisponen a mayor riesgo de presentar malnutrición cuando desarrollan enfermedades graves ${ }^{1,2}$.

Un porcentaje importante de los niños que ingresan a las unidades de cuidados intensivos pediátricos (UCIP) presentan ya malnutrición, entendido este término no sólo como desnutrición sino también como sobrepeso y obesidad. Múltiples estudios recientes, han reportado una prevalencia entre 20 y $47 \%$ de malnutrición en niños críticos, aproximadamente el $13 \%$ de los pacientes de UCIP se han reportado como obesos o con sobrepeso en su admisión; en otros

${ }^{1}$ Médico Pediatra Intensivista de la Unidad de Cuidados Intensivos Pediátricos Hospital Nacional Edgardo Rebagliati Martins- EsSalud, Lima-Perú. Citar como: Liliana Paola Cieza-Yamunaqué. Soporte nutricional en el paciente pediátrico crítico. [Artículo de Revisión].2018;18(4):101-108. (Octubre 2018). DOI 10.25176/RFMH.v18.n4.1737 
estudios la incidencia de malnutrición en el niño crítico varía entre un 40 y un $70 \%{ }^{3,4}$.

La malnutrición en el paciente crítico genera una inadecuada respuesta del organismo contra la enfermedad ocasionando malos resultados clínicos que predispone a infección y desarrollo de fallo multiorgánico, prolongación de estadía en UCI y asistencia ventilatoria mecánica y aumenta la mortalidad $^{3}$.

El soporte nutricional entonces debe formar parte de la estrategia terapéutica desde el inicio en todo niño grave en una UCIP teniendo como objetivos:

- Identificar el riesgo nutricional del paciente crítico.

- Realizar una adecuada valoración nutricional y evaluación de los requerimientos, previniendo subalimentación y sobrealimentación.

- Trazar un plan de nutrición en paciente pediátrico crítico: Proveer nutrientes óptimos, iniciación de soporte nutricional temprano y oportuno, selección de la vía correcta, monitoreo y seguimiento.

En esta revisión el mayor sustento bibliográfico se obtuvo de la última guía de la Sociedad Americana de Cuidados Críticos y A.S.P.E.N. (American Society for Parenteral \& Enteral Nutrition) sobre el Soporte Nutricional del Paciente Pediátrico Crítico del 2017 que actualiza la versión previa del 20095,6.

\section{RESPUESTA METABÓLICA DEL PACIENTE PEDIÁTRICO CRÍTICO}

La injuria entendida como todo tipo de agresión del organismo, puede dividirse en dos categorías, una no mediada por un proceso inflamatorio, cuyo ejemplo es el ayuno y la otra mediada por un proceso inflamatorio, característico del paciente crítico, por ejemplo las infecciones, el choque séptico, el trauma, las quemaduras. Estos dos tipos de injuria generan una respuesta metabólica que es de diferente índole. En el ayuno se inicia una gran cantidad de movilización de proteínas, para garantizar el sustrato glucosa, pero el organismo se adapta, y después de unos días el catabolismo proteico disminuye como una forma de protección frente al compromiso vital que significaría agotar por completo las reservas proteicas, hay un contexto hormonal "de ahorro o hipometabolismo" disminuyendo todas las funciones biológicas para evitar el autoconsumo con bajos niveles de hormonas anabólicas y sin respuesta excesiva de las hormonas catabólicas $^{7,8}$.
En el proceso inflamatorio esta respuesta es exagerada con movilización indefinida de proteínas y el catabolismo proteico tan solo disminuye cuando los mediadores de respuesta inflamatoria han sido controlados; podríamos decir que se presenta un "estrés metabólico", porque no solamente, como en el ayuno, se tiene que sostener las funciones vitales, sino también sostener la respuesta inflamatoria, la función inmunológica y la función tisular, por tanto el gasto es desmesurado, altamente catabólico, hipermetabólicoy son de mayor expresión hormonas contrareguladoras, interleuquinas catabólicas y otros ${ }^{7,8,9}$.

En este contexto el paciente crítico que por su patología presenta una injuria de tipo inflamatoria y que probablemente puede tener también la injuria del ayuno ya sea previo o posterior; presenta una respuesta metabólica muy intensa e importante que puede comprometer la vida del paciente ${ }^{8}$.

Esta respuesta metabólica involucra una serie de reacciones en los hidratos de carbono, aumenta la gluconeogénesis, disminuye la captación de glucosa con resistencia periférica a la insulina, todo ello conlleva a hiperglicemia, a nivel de las grasas hay aumento de la oxidación de grasa y lipólisis, pero la respuesta más importante es a nivel de las proteínas cuyo catabolismo origina una gran degradación de estas debido a que los aminoácidos no sólo se requieren para la gluconeogénesis sino también para la síntesis de proteínas de fase aguda, lo que lleva a gran disminución de proteínas séricas y pérdida de la masa muscular, ocasionando un balance nitrogenado intensamente negativo ${ }^{1-10}$.

Hay dos fases principales en la respuesta metabólica que deben ser entendidas para comprender la importancia del soporte nutricional:

1.Fase Ebb o hipodinámica, la cual se desencadena inmediatamente después de la 12 a 24 horas hasta 48 horas dependiendo de la magnitud y de la severidad del daño, y se caracteriza por una intensa actividad simpática, disminución del volumen sanguíneo, gasto cardiaco, consumo de oxígeno, de la perfusión tisular y gasto energético con lo que se trata de garantizar la circulación y oxigenación de órganos vitales; en esta etapa el objetivo de intervención es estabilizar perfusión y consumo oxígeno con la estabilización hemodinámica y cardiorrespiratoria, que marca fin de etapa Ebb. En esta fase el soporte nutricional tiene poca relevancia ya que el cuerpo se dedica a garantizar la perfusión ${ }^{10,11}$. 
2.Fase flow o hipercatabólica, donde hay una respuesta de hipermetabolismo, desencadenado después del segundo día post-injuria hasta los 7 días posteriores y se caracteriza por un aumento en el gasto cardiaco, consumo de oxígeno, perfusión tisular y gasto energético. Es en esta fase donde el soporte nutricional es muy importante $e^{10,11}$.

Después de las dos primeras fases, hay una etapa de recuperación, anabólica. Pero si hay una nueva injuria, como reinfección o cirugía, estas fases se repetirán indefinidamente ${ }^{1-12}$. Por tanto, el estado metabólico del paciente crítico depende de diversas circunstancias: La etapa de estrés en la que se encuentre, periodo de ayuno, estado nutricional previo, continuidad e intensidad de las injurias presentes, y variabilidad propia de cada organismo.

\section{PLANIFICACIÓN DEL SOPORTE NUTRICIONAL DEL PACIENTE PEDIÁTRICO CRÍTICO}

\section{VALORACIÓN NUTRICIONAL}

La guía ASPEN 2009 recomendaba que al ingresar un niño críticamente enfermo a UCIP se realizara un tamizaje de riesgo nutricional que permitiera la identificación temprana de aquellos niños que están en riesgo de desnutrición durante la estancia hospitalaria y que se beneficiarían de intervenciones nutricionales tempranas. Koen et al hacen una revisión de las herramientas de evaluación de riesgo nutricional en niños, incluyen 6 escalas $^{6-13}$.

- Score de Riesgo Nutricional (NRS)

- Pediatric Score de Riesgo Nutricional (PNR)

- Herramienta de detección para la evaluación de la desnutrición en Pediatría (STAMP)

- Evaluación Subjetiva Global Nutricional (SGNA)

- Pediatric Yorkhill Desnutrición Score (PYMS)

- Herramienta de detección para riesgo de deterioro del estado nutricional (STRONGkids).

De estas herramientas PYMS y STRONGKids fueron los más utilizados en las UCIP y fueron evaluados en múltiples centros de Europa. En la última guía de ASPEN del 2017 se concluye que ninguna de estas herramientas de tamizaje nutricional podrían recomendarse en la práctica clínica. Actualmente, no hay consenso sobre la herramienta de cribado al ingreso 5 .

Para la valoración nutricional se recomienda realizar las puntuaciones z, utilizando las tablas de la Organización Mundial de la Salud (OMS) para peso para la talla $(P / T)$, peso para la edad (P/E) (Si no se dispone de la talla), para expresar el estado nutricional en todo paciente que ingresa a la UCIP5.

La evaluación nutricional antropométrica es simple, reproducible y una herramienta objetiva para la evaluación nutricional del niño enfermo crítico. En la mayoría de los centros terciarios, la realización de las mediciones antropométricas al ingreso se considera como el estándar del cuidado del paciente crítico $^{5-14}$.

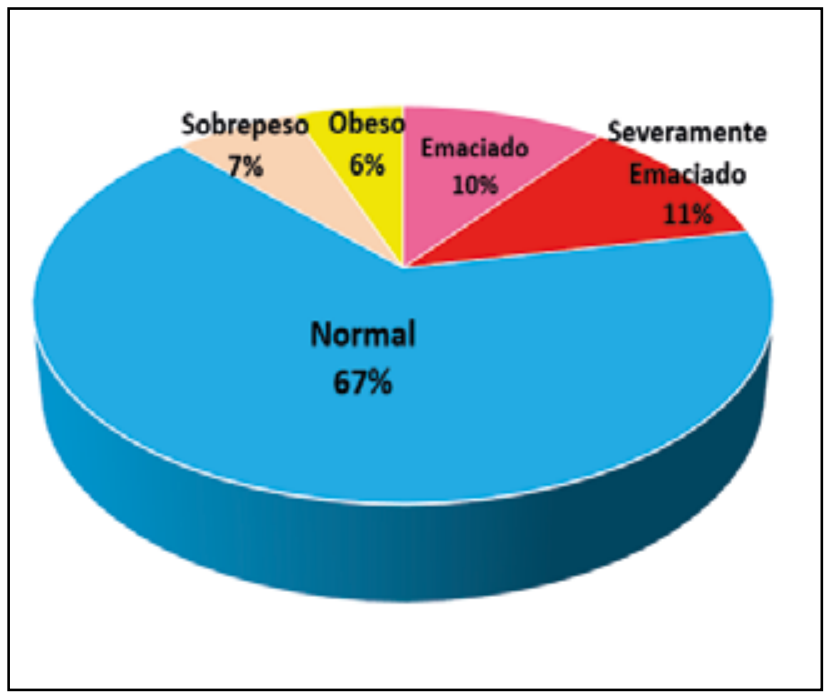

Gráfico 1. Estado nutricional al ingreso UCIP del Hospital Nacional Edgardo Rebagliati Martins (HNERM), 2015-2017.

Los pacientes de la UCIP deben someterse a una evaluación nutricional detallada dentro de las 48 horas de su admisión ${ }^{5}$. Todos los parámetros (antropométricos y bioquímicos) están sujetos a errores e influenciados por cambios en la composición corporal inducidos por factores no nutricionales, en cuanto a la evaluación bioquímica en la fase aguda, los parámetros hormonales y bioquímicos de evaluación nutricional son predominantemente útiles como marcadores de gravedad de la enfermedad y no del estado nutricional per $\mathrm{se}^{6}$

Dentro de la evaluación bioquímica la elevación de la proteína $C$ reactiva $(P C R)$ y la disminución de la prealbúmina son factores predictivos de mortalidad en lactantes durante el posoperatorio. La normalización de los valores altos de PCR indicaría un retorno al anabolismo ${ }^{10-14}$.

\section{CÁLCULO DE REQUERIMIENTOS ENERGÉTICOS RECOMENDADOS PARA NIÑOS CRÍTICAMENTE ENFERMOS}

Para calcular los requerimientos energéticos se utiliza el gasto energético en reposo (GER). El GER se define como la cantidad de calorías requeridas por el cuerpo en reposo durante un período de 24 horas y representa 
el $70 \%$ del gasto energético total; constituye la suma de tasa metabólica basal (TMB) más la termogénesis endógena producida por los alimentos ${ }^{15-11}$.

El patrón de oro para la medición del GER es la calorimetría indirecta $(\mathrm{Cl})$ para determinar los requisitos de energía y guiar la prescripción del objetivo energético diario. $\mathrm{La} \mathrm{Cl}$, estima el GER a partir de la medición de los gases respiratorios inspirados y espirados, es el mejor método para valorar el consumo calórico individual. Sin embargo, los monitores de $\mathrm{Cl}$ no están al alcance de la mayoría de centros y los resultados pueden alterarse si el paciente tiene una fracción inspirada de oxígeno (FIO2) mayor del 60\%, una fuga del tubo endotraqueal mayor del 10\% y durante la oxigenación por membrana extracorpórea y las técnicas de depuración extrarrenal continuas venovenosas ${ }^{16}$.
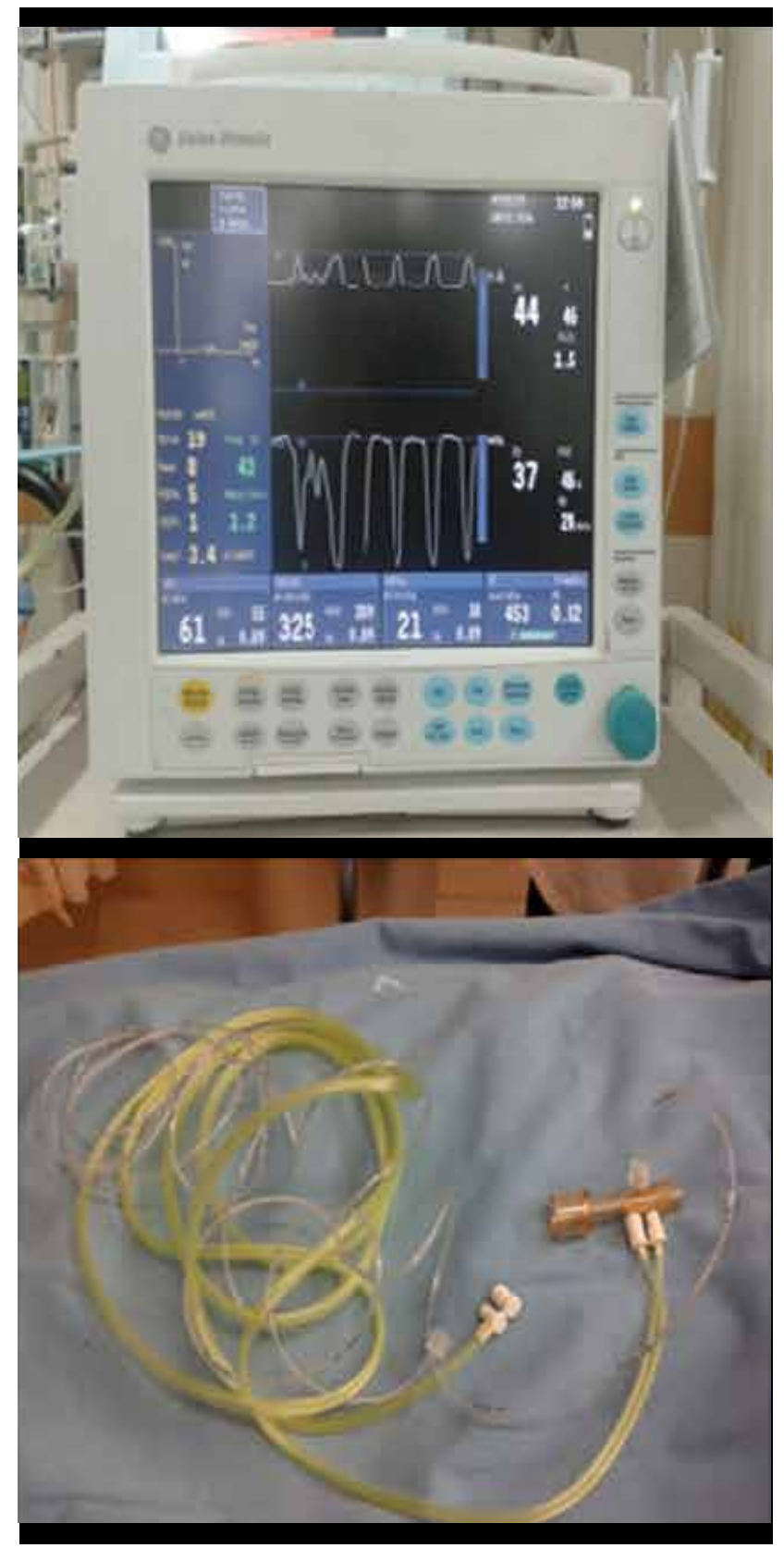

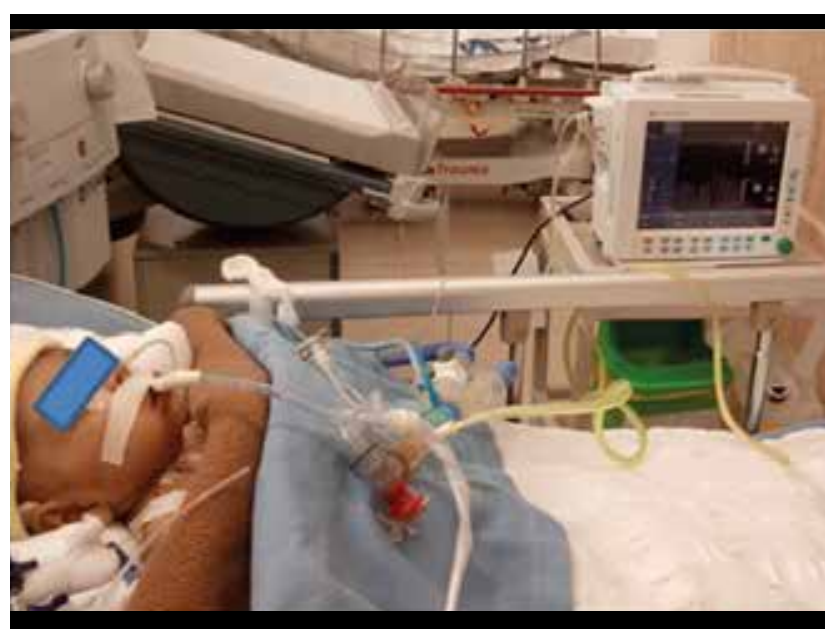

Figura 1. Calorímetro en el paciente pediátrico crítico.

Si no es factible hacer la medición del GER con calorimetría, se debe utilizar las fórmulas para calcular el GER de Schofield o las de la OMS "sin" la adición de factores de estrés para estimar el GER .El HarrisBenedict y las RDAs, no debe usarse para niños críticamente enfermos ${ }^{5-3}$.

\begin{tabular}{|c|c|}
\hline \multicolumn{2}{|c|}{ WHO equation (Kcal/day) } \\
\hline \multirow{2}{*}{$<3$ yrs: } & Boy: $(60.9 \times W t)-54$ \\
\hline & Girl: $(61 \times W t)-51$ \\
\hline \multirow[t]{2}{*}{ 3-10 yrs: } & Boy: $(22.7 \times W t)+495$ \\
\hline & Girl: $(22.5 \times W t)+499$ \\
\hline \multicolumn{2}{|c|}{$10-18$ yrs: Boy: $(17.5 \times W t)+651$} \\
\hline \multicolumn{2}{|c|}{ Girl: $(12.2 \times W t)+746$} \\
\hline \multicolumn{2}{|c|}{ Schofield equations $(\mathrm{MJ} /$ day $)(1 \mathrm{kcal}=4.186 \mathrm{~kJ})$} \\
\hline \multirow[t]{2}{*}{$<3$ yrs: } & Boy: $(0.0007 \times \mathrm{Wt})+(6.349 \times \mathrm{Ht})-2.584$ \\
\hline & Girl: $(0.068 \times \mathrm{Wt})+(4.281 \times \mathrm{Ht})-1.730$ \\
\hline \multirow[t]{2}{*}{ 3-10 yrs: } & Boy: $(0.082 \times W t)+(0.545 \times H t)+1.736$ \\
\hline & Girl: $(0.071 \times W t)+(0.677 \times H t)+1.553$ \\
\hline \multirow[t]{2}{*}{$10-18$ yrs: } & Boy: $(0.068 \times W t)+(0.574 \times H t)+2.157$ \\
\hline & Girl: $(0.035 \times W t)+(1.948 \times H t)+0.837$ \\
\hline
\end{tabular}

W: peso en Kilos, $\mathrm{Ht}$ : Talla en centímetros.

Figura 2. Ecuaciones para GER Schofield y OMS.

Tabla 1 . Cálculo aproximado de GER según grupo etáreo.

\begin{tabular}{|c|c|c|c|c|c|}
\hline & $<1 \mathrm{yr}$ & 1-2yrs & $2-5 y r s$ & $6-12 y r s$ & $>13 y r s$ \\
\hline & $\begin{array}{c}\mathrm{kcal} / \mathrm{kg} / \\
\text { day }\end{array}$ & $\begin{array}{c}\mathrm{kcal} / \mathrm{kg} / \\
\text { day }\end{array}$ & $\begin{array}{c}\mathrm{kcal} / \mathrm{kg} / \\
\text { day }\end{array}$ & $\begin{array}{l}\mathrm{kcal} / \mathrm{kg} / \\
\text { day }\end{array}$ & $\mathrm{kcal} / \mathrm{kg} / \mathrm{day}$ \\
\hline Schofield & $51-54$ & $56-57$ & $45-57$ & $33-45$ & $26-29$ \\
\hline WHO REE & 68 & 57 & 53 & 38 & 28 \\
\hline
\end{tabular}

\section{WHO REE: GER POr OMS}

Se debería lograr al menos $70-80 \%$ del GER al final de la primera semana en el paciente crítico, debido a que una subalimentación provocaría una falla en la cicatrización de heridas, mayor riesgo de infecciones, mayores secuelas en los pacientes, deterioro cognitivo y aumento de la mortalidad y estadía en UCIP. Podría llegarse al $100 \%$ del GER en los primeros 7 días sin 
traspasar este valor debido a que la hiperalimentación ocasiona hiperglicemia,hipertrigliceridemia, colestasis o esteatosis hepática, falla respiratoria, uremia y prolongación de la estancia en UCIP5.

\section{APORTE DE MACRONUTRIENTES}

El aporte de proteínas es lo más importante cuando se aporta macronutrientes, debido a la respuesta metabólica descrita anteriormente en la que el balance negativo de proteínas puede resultar en pérdida de la masa muscular magra, que se ha asociado con malos resultados en pacientes críticamente enfermos. Es por ello que la guía ASPEN 2017 pone énfasis y recomienda un consumo mínimo de proteínas de 1,5 g / kg / día. En lactantes y niños pequeños, la ingesta óptima de proteínas necesaria para lograr un equilibrio positivo de proteínas puede ser mucho mayor. En diversos artículos un mayor consumo de proteínas no se asoció con un retraso en la recuperación renal en pacientes críticos con insuficiencia renal aguda ${ }^{17,18}$.

Es importante la provisión de proteína al inicio del curso de la enfermedad crítica para alcanzar las metas proteícas y promover balance de nitrógeno positivo ${ }^{5-17}$.

Tabla 2. Requerimientos proteicos estimados según grupo etáreo.

\begin{tabular}{cccccc}
\hline & $<1$ yr & $1-2 y r s$ & $2-3 y r s$ & $3-12 y r s$ & $>13 y r s$ \\
& $\mathbf{g} / \mathbf{k g} /$ day & $\mathbf{g} / \mathbf{k g} /$ day & $\mathbf{g} / \mathbf{k g} /$ day & $\mathbf{g} / \mathbf{k g} /$ day & $\mathbf{g} / \mathbf{k g} /$ day \\
\hline ASPEN & $2.0-3.0$ & $2.0-3.0$ & $1.5-2.0$ & $1.5-2.0$ & 1.5 \\
\hline
\end{tabular}

Una vez alcanzada la meta proteica, asegurar el aporte de hidratos de carbono y de grasas como fuente de energía, tiene efectos beneficiosos sobre la síntesis proteica neta y el balance general de proteínas. La relación de calorías no proteica por gramos de nitrógeno en el paciente crítico es de 80 a $120^{5}$.

Según la guía ASPEN 2017, no hay evidencia de alta calidad para dar recomendaciones acerca del aporte de los demás macronutrientes en niños con enfermedades críticas, sólo consenso de expertos ${ }^{5,6}$.

Con respecto al aporte de hidratos de carbono, si tomamos en cuenta su metabolismo debemos considerar que la glucosa es necesaria para reparar tejidos lesionados $y$, además, es el sustrato esencial usado por el cerebro, los eritrocitos y la médula renal. Los requerimientos de glucosa deben administrarse según la edad. Las variaciones son perjudiciales, la hiperglucemia es un factor que aumenta la morbimortalidad. La hipoglucemia puede causar daño neurológico, por lo tanto, debe ser rápidamente diagnosticada y $\operatorname{tratada}^{19}$.

El recambio en el metabolismo lipídico está acelerado, esta demanda aumentada se asocia a depósitos de grasa disminuidos y podría aparecer rápidamente déficit de ácidos grasos esenciales, trombocitopenia y aumento de susceptibilidad a las infecciones. Los requerimientos de lípidos se inician a $1 \mathrm{~g} / \mathrm{kg} /$ día y se avanza cada 24 h hasta $2-4 \mathrm{~g} / \mathrm{kg} /$ día (aportes más altos en niños más pequeños) con monitoreo de trigliceridemia, sin sobrepasar concentraciones del $30 \%-40 \%$ del total de calorías ${ }^{19}$.

\section{ELECCIÓN DE VÍA A UTILIZAR EN EL PACIENTE PEDIÁTRICO CRÍTICO}

Es necesario evaluar la función gastrointestinal al ingreso a UCIP para poder determinar así la vía a utilizar $^{20}$.

\section{Nutrición enteral (NE)}

Es el modo preferido de entrega de nutrientes en el niño críticamente enfermo si el tracto gastrointestinal está funcionante.(20)

La NE respeta las funciones digestivas, tiene menos riesgos de complicaciones, favorece el trofismo de la mucosa, estimula mecanismos neuroendocrinos (intraluminales y extraluminales), no requiere técnicas de asepsia complejas, puede contribuir al mantenimiento de la respuesta inmunitaria, disminuye la posibilidad de translocación bacteriana, puede ser utilizada en centros de menor complejidad y es menos costosa. Así mismo, es segura en niños críticamente enfermos con diagnósticos médicos y quirúrgicos, también en los que reciben medicaciones vasoactivas $^{20}$.

La ruta enteral debe iniciarse tempranamente una vez estabilizado el paciente dentro de las 24-48 horas de la admisión a la UCIP y lograr por lo menos un 70\% del GER en la primera semana crítica 5 .

Las barreras comunes a la NE en la UCIP incluyen retraso en la iniciación, interrupciones debidas a la percepción de intolerancia y ayuno prolongado en torno a los procedimientos, por ello se recomienda minimizar las interrupciones 5 .

La intolerancia a la NE es el principal factor que limita el aporte enteral de nutrientes y podría incrementar la dependencia de la nutrición parenteral (NP) para alcanzar las metas nutricionales. Los criterios más 
comúnmente usados para percibir intolerancia son volumen residual gástrico (VRG) alto, distensión abdominal, diarrea y vómitos, y se deben utilizar en conjunto para monitorear la tolerancia a la NE. El valor del monitoreo del VRG es dudoso y podría ser una barrera para la provisión de NE, pues su medición como guía de la alimentación o como marcador de riesgo de aspiración es cuestionable. Por tanto, la decisión de interrumpir la NE no debería basarse sólo en un valor arbitrario de VRG, este debería evaluarse individualmente después de haber considerado los otros signos de intolerancia antes mencionados ${ }^{20}$.

\section{Técnica de administración de la nutrición enteral}

Se recomienda conseguir en $48 \mathrm{~h}$ por lo menos el $25 \%$ de los requerimientos nutricionales, no habiéndose demostrado diferencia estadísticamente significativa entre administración intermitente e infusión continua 5 .

1.Nutrición intermitente: Se puede iniciar con el $25 \%$ de la meta calórica dividida entre el número de tomas deseadas, incrementando el aporte de fórmula en un $25 \%$ por día, dividiendo la cantidad del volumen por partes iguales entre el número de tomas.(20)

2. Nutrición enteral en Infusión continua: Iniciar con una fórmula a $1-2 \mathrm{ml} / \mathrm{kg} / \mathrm{h}$ y progresar $0.5-1 \mathrm{ml} / \mathrm{Kg} /$ hora cada 6-24 horas.

$>25 \mathrm{Kg}$ se podría iniciar con $25 \mathrm{ml} /$ hora y progresar cada 4 horas $25 \mathrm{ml} /$ hora $^{20}$.

\section{Uso de sonda nasogastrica o transpilórica}

Los datos existentes son insuficientes para hacer recomendaciones universales con respecto al sitio óptimo para entregar NE a los niños críticamente enfermos. Diversos estudios sugieren que la ruta gástrica sea el sitio preferido para el inicio de la NE por ser más fisiológica. La sonda transpilórica (STP) puede ser utilizada en pacientes incapaces de tolerar alimentación por sonda nasogástrica (SNG) o aquellos con alto riesgo de aspiración. El uso de la STP puede mejorar la ingesta calórica comparada con la gástrica ya que disminuye las interrupciones relacionadas con procedimientos, aunque otros estudios difieren pues encontraron retrasos al inicio de la NE por ser la colocación de STP, de mayor dificultad que la SNG.(5)

Un enfoque algorítmico nos ayuda a instaurarla NE del paciente pediátrico crítico para avanzar NE como se muestra en la figura 6.

\section{Nutrición parenteral (NP)}

Las indicaciones de NP son: Paciente en el cual el tracto gastrointestinal no pueda ser utilizado, paciente pediátrico que requiera ayuno absoluto igual o superior de 5 días o antes si es un niño desnutrido, en aquellos en los que no sea posible la vía enteral, con una ingesta inferior al $50 \%$ de sus necesidades durante los 7 días o antes en el desnutrido. Su administración debe ser por vía central exclusiva, de preferencia catéteres epicutáneos y si no es posible catéter venoso central sólo para este fin ${ }^{21}$.

La NP suplementaria, es la que se da en conjunto con la $\mathrm{NE}$, cuando la vía enteral no logra conseguir la meta calórica. Esta debe retrasarse hasta 1 semana después de la admisión de la UCIP en pacientes con estado nutricional basal normal y bajo riesgo de deterioro nutricional. En pacientes que están gravemente desnutridos o en riesgo del deterioro nutricional la NP puede iniciarse antes.

La NP debe mantenerse hasta que se consiga una adecuada transición a la $\mathrm{NE}$, es decir, cuando dichos aportes alcancen $2 / 3$ de los requerimientos nutricionales estimados ${ }^{5-21}$.

\section{INMUNONUTRICIÓN}

Basado en la evidencia pediátrica disponible no se recomienda de rutina la inmunonutrición ${ }^{5}$.

\section{SEGUIMIENTO E INTERVENCIÓN DE EQUIPO MULTIDISCIPLINARIO}

Un equipo de soporte nutricional (ESN) en UCIP optimizaría las prácticas nutricionales permitiendo cumplir los protocolos, mejorar la disponibilidad de nutrientes, acortar el tiempo para alcanzar el objetivo nutricional, incrementar la NE y disminuir el uso de NP. La utilización de registros de salud electrónicos personalizados pueden ayudar mejorar la vigilancia del soporte nutricional.

Los pacientes que están en riesgo de deterioro nutricional durante la hospitalización, deben ser reevaluados al menos una vez por semana durante la su estancia en UCIP5.

\section{CONCLUSIÓN}

El soporte nutricional del paciente pediátrico crítico es trascendental para mejorar su morbimortalidad, y es necesaria una planificación adecuada de dicho soporte para obtener resultados óptimos. El soporte nutricional es una importante estrategia terapéutica en todo niño grave. 


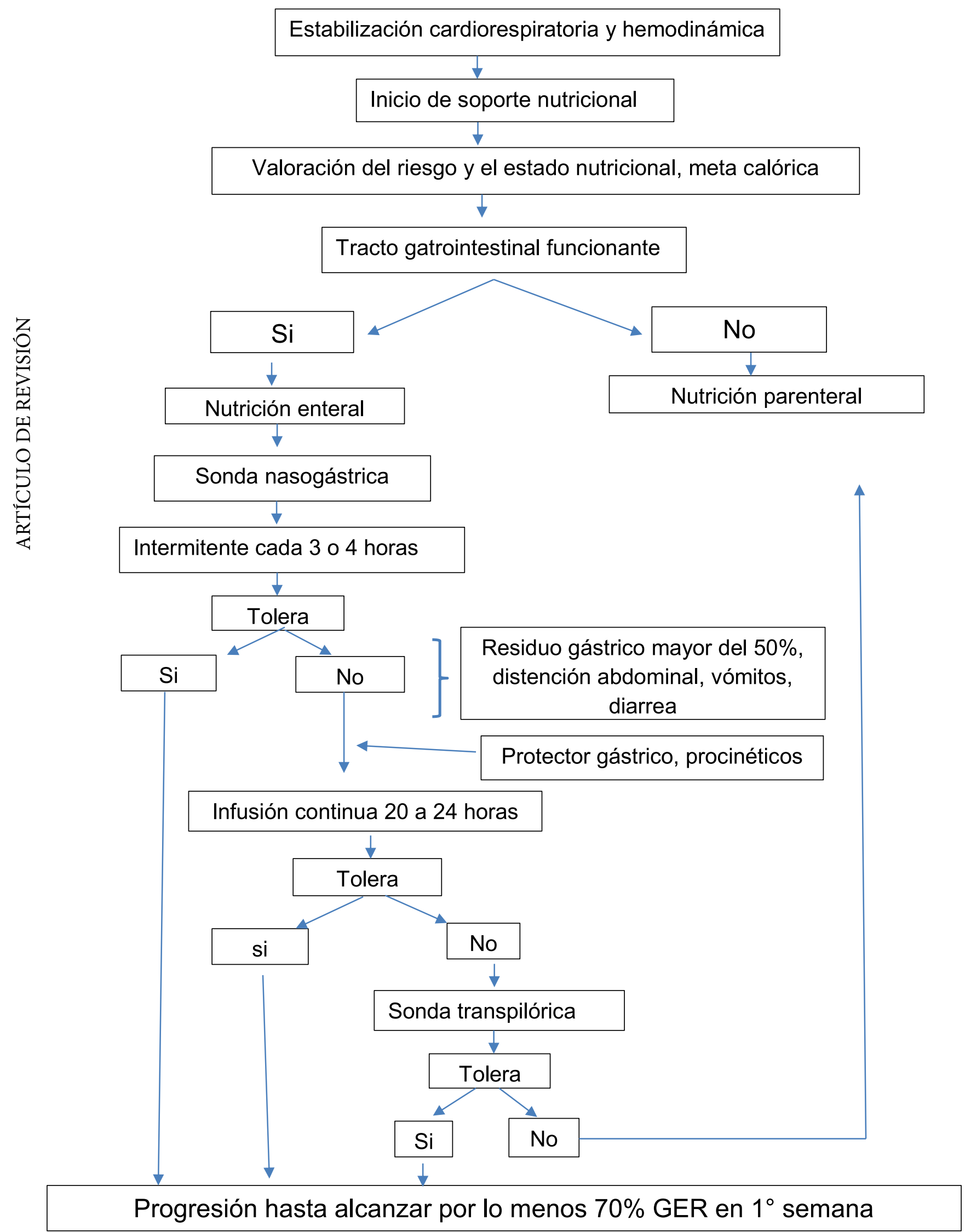

Figura 3. Algorítmico de instauración de NE o NP. 
Contribuciones de autoría: La autora participó en la idea, recolección de la información, redacción y revisión del presente manuscrito.

Financiamiento: Autofinanciado.

Conflictodeinterés:Laautoradeclaranotenerconflictos de interés con la publicación de este manuscrito.

Recibido: 20 de junio del 2018

Aprobado: 30 de agosto del 2018

Correspondencia: Liliana Paola Cieza Yamunaqué

Dirección: Jr. Belisario Flores 238 Dpto 301 - Lince. Lima 14, Lima-Perú

Celular: +51-990452547

Correo:liciya04@hotmail.com

\section{REFERENCIAS BIBLIOGRÁFICAS}

1. Preiser J-C, Van Zanten A, Berger MM, Biolo G, Casaer MP, Doig GS, et al. Metabolic and nutritional support of critically ill patients: consensus and controversies. Crit Care. 2015;19(1):35.

2. Verger J. Nutrition in the Pediatric Population in the Intensive Care Unit. Crit Care Nurs Clin North Am. 2014 Jun;26(2):199-215.

3. Martinez EE, Mehta NM. The science and art of pediatric critical care nutrition. Curr Opin Crit Care. 2016 Aug;22(4):316-24.

4. López-Herce Cid J. La nutrición del niño en estado crítico. An Pediatría. 2009 Jul;71(1):1-4.

5. Mehta NM, Skillman HE, Irving SY, et al. Guidelines for the Provision and Assessment of Nutrition Support Therapy in the Pediatric Critically III Patient: Society of Critical Care Medicine and American Society for Parenteral and Enteral Nutrition. J Parenter Enter Nutr. 2017 Jul 2;41(5):706-42. doi 10.1177/0148607117711387

6. Mehta NM, Compher C. A.S.P.E.N. Clinical Guidelines: Nutrition Support of the Critically III Child. J Parenter Enter Nutr. 2009 May 27;33(3):260-76. doi/10.1177/0148607109333114

7. Fraipont V, Preiser J-C. Energy Estimation and Measurement in Critically III Patients. J Parenter Enter Nutr. 2013 Nov 10;37(6):705-13. doi/10.1177/0148607113505868

8. Cruz R. Respuesta Metabólica a la Injuria Grave. Rev Nutr Clin. 2012;6(19):1011-9.

9. Skillman HE, Mehta NM. Nutrition therapy in the critically ill child. Curr Opin Crit Care. 2012 Apr;18(2):192-8.

10. Joosten KFM, Kerklaan D, Verbruggen SCAT. Nutritional support and the role of the stress response in critically ill children. Curr Opin Clin Nutr Metab Care. 2016 Mar;1.

11. De Cosmi V, Milani GP, Mazzocchi A, D'Oria V, Silano M, Calderini E, et al. The Metabolic Response to Stress and Infection in Critically III Children: The Opportunity of an Individualized Approach. Nutrients. 2017 Sep 18;9(9):1032.
12. Ramirez Santos. Respuesta Metabólica al Trauma. MEDICRIT Rev Med Crítica. Volumen 5:130-3.

13. Joosten KFM, Hulst JM. Nutritional screening tools for hospitalized children: Methodological considerations. Clin Nutr. 2014 Feb;33(1):1-5

14. Mehta NM, Bechard LJ, Cahill N, Wang M, Day A, Duggan CP, et al. Nutritional practices and their relationship to clinical outcomes in critically ill children-An international multicenter cohort study*. Crit Care Med. 2012 Jul;40(7):2204-11.

15. Beggs MR, Garcia Guerra G, Larsen BMK. Do PICU patients meet technical criteria for performing indirect calorimetry? Clin Nutr ESPEN. 2016 Oct;15:80-4.

16. Sion-Sarid R, Cohen J, Houri Z, Singer P. Indirect calorimetry: A guide for optimizing nutritional support in the critically ill child. Nutrition. 2013 Sep;29(9):1094-9.

17. Sethi SK, Maxvold N, Bunchman T, Jha P, Kher V, Raina R. Nutritional management in the critically ill child with acute kidney injury: a review. Pediatr Nephrol. 2017 Apr 20;32(4):589-601.

18. Sabatino A, Regolisti G, Maggiore U, Fiaccadori E. Protein/Energy Debt in Critically III Children in the Pediatric Intensive Care Unit: Acute Kidney Injury As a Major Risk Factor. J Ren Nutr. 2014 Jul;24(4):209-18.

19. Galera-Matinez. Actualización en el soporte nutricional del paciente pediátrico críticamente enfermo. Acta Pediátrica Española. 2017;75:117-23.

20. Zamberlan P, Delgado AF, Leone C, Feferbaum R, Okay TS. Nutrition Therapy in a Pediatric Intensive Care Unit. J Parenter Enter Nutr. 2011 Jul 24;35(4):523-9.

21. Mantegazza C, Landy N, Zuccotti G V., Köglmeier J. Indications and complications of inpatient parenteral nutrition prescribed to children in a large tertiary referral hospital. Ital J Pediatr. 2018 Dec 8;44(1):66. 